\title{
ON LOCAL EMBEDDING PROPERTIES OF INJECTORS OF FINITE SOLUBLE GROUPS
}

\author{
STEPHANIE REIFFERSCHEID \\ (Received 25 February 2002; revised 3 January 2003) \\ Communicated by E. O'Brien
}

\begin{abstract}
In the present paper we consider Fitting classes of finite soluble groups which locally satisfy additional conditions related to the behaviour of their injectors. More precisely, we study Fitting classes $\mathfrak{X}, \mathfrak{F}$, $1 \neq \mathfrak{X} \subseteq \mathfrak{F}$, such that an $\mathfrak{X}$-injector of $G$ is, respectively, a normal, (sub)modular, normally embedded, system permutable subgroup of $G$ for all $G \in \mathfrak{F}$.

Locally normal Fitting classes were studied before by various authors. Here we prove that some important results-already known for normality - are valid for all of the above mentioned embedding properties. For instance, all these embedding properties behave nicely with respect to the Lockett section. Further, for all of these properties the class of all finite soluble groups $G$ such that an $\mathfrak{X}$-injector of $G$ has the corresponding embedding property is not closed under forming normal products, and thus can fail to be a Fitting class.
\end{abstract}

2000 Mathematics subject classification: primary 20D10, $20 \mathrm{~F} 17$.

\section{Introduction}

In the investigation of Fitting classes of finite soluble groups it seems natural to restrict oneself to Fitting classes satisfying additional conditions related to the behaviour of their injectors. For instance, Blessenohl and Gaschütz [1], Hauck and Kienzle [7], Lockett [8] and Doerk and Porta [5] studied non-trivial Fitting classes whose injectors are respectively normal, (sub)modular, normally embedded and system permutable subgroups of $G$ in each group $G \in \mathfrak{S}$. These investigations can be generalized by considering non-trivial Fitting classes $\mathfrak{X}$ and $\mathfrak{F}$ of finite soluble groups such that $\mathfrak{X}$ is contained in $\mathfrak{F}$ and an $\mathfrak{X}$-injector of $G$ satisfies a given embedding property e in $G$

(C) 2004 Australian Mathematical Society $1446-7887 / 04 \$ A 2.00+0.00$ 
for every group $G \in \mathfrak{F}$ (thus by investigating these embedding properties "locally" in $\mathfrak{F}$, the global case being $\mathfrak{F}=\mathfrak{S}$ ). In this situation we call $\mathfrak{X}$ an $\mathfrak{F}_{e}$-class, and we use $Y_{\mathrm{e}}(\mathfrak{X})$ to denote the class of all groups $G \in \mathfrak{S}$ such that an $\mathfrak{X}$-injector of $G$ satisfies a given embedding property e in $G$. Locally normal Fitting classes $\left(\mathfrak{F}_{\mathrm{n}}\right.$-classes) were studied before by various authors (see for instance Hauck [6], Reifferscheid [12]), and so in the present paper we will concentrate on the-in part considerably weakerembedding properties (sub)modularity ((s)mod), normal embedding (ne) and system permutability (p). Since one of the first results to emerge is that the class $Y_{\bmod }(\mathfrak{X})$ is not closed under direct products, and consequently that the concepts of locally modular and locally normal Fitting classes coincide, in the following we consider local submodularity instead of local modularity. Studying the above relations we will frequently concentrate on results valid for locally normal Fitting classes. For instance, local normality is a property of the corresponding Lockett sections, that is, $\mathfrak{X}$ is normal in $\mathfrak{F}$ precisely when $\mathfrak{X}^{*}$ is normal in $\mathfrak{F}^{*}$ (see $[6,4.8]$ or $[4, X, 3.3]$ ), and we will see that this is also true for the other embedding properties.

Further, the class $Y_{n}(\mathfrak{X})$ of all groups $G$ such that an $\mathfrak{X}$-injector is normal in $G$ is not-in general-closed under products of normal subgroups (see [6, 3.2]), and thus can fail to be a Fitting class. Here, too, an analogous result is true for the other embedding properties. Even more-and this is in contrast to normality- the class $Y_{p}(\mathfrak{X})$ is closed under forming products of normal subgroups if and only if it coincides with the class $\mathfrak{S}$ of all (finite and soluble) groups.

As dual to the investigation of $\mathrm{Y}_{\mathrm{e}}(\mathfrak{X})$, the question on the intersection of $\mathfrak{F}_{\mathrm{e}^{-}}$ classes-and thus on the existence of a unique minimal $\mathfrak{F}_{\mathrm{e}}$-class-naturally arises. While it is known at least for Fischer classes $\mathfrak{F}$ that the (non-trivial) intersection of $\mathfrak{F}_{\mathfrak{n}}$-classes is again an $\mathfrak{F}_{\mathfrak{n}}$-class (see $[6,4.12]$ ), this question is open in general for the embedding properties submodularity and normal embedding even in the case that all classes under consideration are Fischer classes or, stronger, subgroup-closed Fitting classes. In the case of local permutability a negative answer is known for $\mathfrak{F}=\mathfrak{S}([4$, IX, 3.14]).

Since-as is well known-local normality between Lockett classes behaves nicely with respect to the regular wreath product, for the important classes $\mathfrak{F}=\mathfrak{N}^{i+1}, i \in \mathbb{N}$, and $\mathfrak{F}=\mathfrak{S}_{\pi_{1}} \ldots \mathfrak{S}_{\pi_{r}}, \pi_{1}, \ldots, \pi_{r}$ sets of primes, the existence of a unique minimal $\mathfrak{F}_{n}$-class can be shown $([10,2.3],[11,3.1 .18])$. We will see that local submodularity too behaves well with respect to regular wreath products, and consequently that an analogous result is valid in this case. Furthermore, it turns out that for these classes the smallest $\mathfrak{F}_{\mathfrak{n}}$-class and the smallest $\mathfrak{F}_{\text {smod }}$-class coincide. That the concept of local submodularity is very close to the concept of local normality is also highlighted by the fact that these concepts coincide for subgroup-closed Fitting classes, hence for subgroup-closed Fitting classes $\mathfrak{X}$ and $\mathfrak{F}$ it holds that $\mathfrak{X}$ is an $\mathfrak{F}_{\mathrm{n}}$-class precisely when $\mathfrak{X}$ is an $\mathfrak{F}_{\text {smod }}$-class. 


\section{Notations and preliminaries}

All groups considered in this paper are assumed to be finite and soluble.

Except for denoting the regular wreath product of $G$ and $H$ by $G$ ? $H$ and its base group by $G^{*}$, we shall adhere to the notation used in [4]. This book is also the main reference for all results concerning classes of groups.

Recall that a class $\mathfrak{F}$ of groups is called a Fitting class if it is closed under taking subnormal subgroups $\left(s_{n} \mathfrak{F}=\mathfrak{F}\right)$ and products of normal subgroups $\left(N_{0} \mathfrak{F}=\mathfrak{F}\right)$. The latter implies that in each group $G$ there exists a unique normal subgroup being maximal among all normal subgroups of $G$ contained in $\mathfrak{F}$, the so-called $\mathfrak{F}$-radical $G_{\mathfrak{F}}$ of $G$. By a well-known result of Fischer, Gaschütz and Hartley (see [4, IX, 1.4]), Fitting classes of finite soluble groups are exactly those classes $\mathfrak{F}$ such that in every (finite soluble) group $G$ there exists a unique conjugacy class of so-called $\mathfrak{F}$-injectors of $G$, subgroups $U$ of $G$ such that $U \cap N$ is $\mathfrak{F}$-maximal in $N$, that is, maximal among all subgroups of $N$ contained in $\mathfrak{F}$, for all subnormal subgroups $N$ of $G$. The set of all $\mathfrak{F}$-injectors of $G$ is denoted by $\operatorname{Inj}_{\mathfrak{F}}(G)$.

Let $\mathfrak{X}, \mathfrak{F}$ be Fitting classes. Recall that $\mathfrak{X}$ is said to be normal in $\mathfrak{F}$ ( $\mathfrak{F}$-normal, $\mathfrak{F}_{\mathrm{a}}$-class) if $1 \neq \mathfrak{X} \subseteq \mathfrak{F}$ and an $\mathfrak{X}$-injector of $G$ is a normal subgroup of $G$ for every $G \in \mathfrak{F}$. Further, we use $\mathrm{Y}_{\mathrm{n}}(\mathfrak{X})$ to denote the class of all groups $G$ such that an $\mathfrak{X}$-injector of $G$ is a normal subgroup of $G$.

As usual we use $\mathfrak{S}, \mathfrak{S}_{\pi}, \mathfrak{N}, \mathfrak{N}_{\pi}$ to denote respectively the class of all (finite soluble) groups, the class of all (finite soluble) $\pi$-groups, the class of all (finite) nilpotent groups and the class of all (finite) nilpotent $\pi$-groups, where $\pi$ denotes a set of primes.

Recall further that the characteristic Char( $\mathfrak{F})$ of a Fitting class $\mathfrak{F}$ consists of all primes $p$ such that $Z_{p} \in \mathfrak{F}$, that this set coincides with the set $\pi(\mathfrak{F})=\bigcup_{G \in \mathfrak{F}} \pi(G)$, where $\pi(G)$ denotes the set of all prime divisors of $|G|$, and that $\mathfrak{N}_{\pi(\mathfrak{F})} \subseteq \mathfrak{F} \subseteq \mathfrak{S}_{\pi(\mathfrak{F})}$ holds true (see $[4, \mathrm{IX}, 1.7,1.9]$ ).

Further, we use for a Fitting class $\mathfrak{X}$

$$
l(\mathfrak{X}):= \begin{cases}\min \left\{r \in \mathbb{N} \mid \mathfrak{X} \subseteq \mathfrak{N}^{r}\right\} & \text { if it exists, } \\ \infty & \text { otherwise }\end{cases}
$$

to denote the nilpotent length of $\mathfrak{X}$. (Note that the Fitting class product $\mathfrak{X Y}$ of Fitting classes $\mathfrak{X}$ and $\mathfrak{Y}$ denotes the Fitting class consisting of all groups $G$ such that $G / G_{\mathfrak{X}} \in \mathfrak{Y}$, and set $\mathfrak{X}^{0}=1$ the trivial class, and $\mathfrak{X}^{i}=\mathfrak{X}^{i-1}$ for $i \in \mathbb{N}$.)

Lockett ([8]) associated to each Fitting class $\mathfrak{F}$ the class $\mathfrak{F}^{*}$ which by definition consists of all groups $G$ such that $(G \times G)_{\mathfrak{F}}$ is subdirect in $G \times G$. We collect some properties of this class in the following theorem (see [4, X, Section 1]):

THEOREM 2.1. Let $\mathfrak{F}$ be a Fitting class and $G$ be a group. 
(a) $\mathfrak{F}^{*}$ is a Fitting class.

(b) $(G \times G)_{\mathfrak{F}}=\left(G_{\mathfrak{F}} \times G_{\mathfrak{F}}\right)\left\langle\left(g, g^{-1}\right) \mid g \in G_{\mathfrak{F}}\right\rangle$.

(c) $G_{\mathfrak{F}} / G_{\mathfrak{F}}$ is abelian.

(d) If $V \in \operatorname{Inj}_{\mathfrak{F}^{*}}(G)$, then $V_{\mathfrak{F}}$ is an $\mathfrak{F}$-injector of $G$.

(e) Let $\left\{\mathfrak{F}_{i}\right\}_{i \in I}$ be a family of Fitting classes. Then $\left(\bigcap_{i \in l} \mathfrak{F}_{i}\right)^{*}=\bigcap_{i \in I} \mathfrak{F}_{i}^{*}$.

A Fitting class $\mathfrak{F}$ is called a Lockett class if $\mathfrak{F}=\mathfrak{F}^{*}$. For each Fitting class $\mathfrak{F}$, we define $\mathfrak{F}_{*}=\bigcap\left\{\mathfrak{X} \mid\right.$ Fitting class and $\left.\mathfrak{X}^{*}=\mathfrak{F}^{*}\right\}$ and call $\left\{\mathfrak{X} \mid \mathfrak{X}^{*}=\mathfrak{F}^{*}\right\}=\left\{\mathfrak{X} \mid \mathfrak{F}_{*} \subseteq\right.$ $\mathfrak{X} \subseteq \mathfrak{F}^{*}$ \} the Lockett section of $\mathfrak{F}$.

By definition, each Q-closed Fitting class (see below) is a Lockett class. In particular, $\mathfrak{S}, \mathfrak{S}_{\pi}, \mathfrak{N}, \mathfrak{N}_{\pi}$ are Lockett classes.

Radicals and injectors of Lockett classes behave nicely with respect to direct products (see [4, X, 1.9, 1.33]):

THEOREM 2.2. Let $\mathfrak{F}$ be a Lockett class and $G, G_{1}, G_{2}$ be groups.

(a) $\left(G_{1} \times G_{2}\right)_{\mathfrak{F}}=\left(G_{1}\right)_{\mathfrak{F}} \times\left(G_{2}\right)_{\mathfrak{F}}$.

(b) Let $V$ be an $\mathfrak{F}$-injector of $G$. Then $V=\left(V \cap G_{1}\right) \times\left(V \cap G_{2}\right)$, in particular $V=V_{1} \times V_{2}$ where $V_{i} \in \operatorname{Inj}_{\mathfrak{F}}\left(G_{i}\right)$ for $i=1,2$, and every subgroup of this form is an F-injector of $G_{1} \times G_{2}$.

If $\mathfrak{F}$ is an $\mathrm{N}_{0}$-closed class of groups such that $H \leq G \in \mathfrak{F}, G /$ Core $_{G}(H) \in \mathfrak{N}$ implies $H \in \mathfrak{F}$, then $\mathfrak{F}$ is called a Fischer class, and $\mathfrak{F}$ is a subgroup-closed Fitting class if it is closed under taking subgroups $(s \mathfrak{F}=\mathfrak{F})$.

Recall that by a deep result of Bryce and Cossey ([2, Theorem 1], [3, Theorem 1.1]) subgroup-closed Fitting classes of finite soluble groups are saturated formations. Consequently, if $\mathfrak{F}$ is a subgroup-closed Fitting class then

$$
\begin{aligned}
\mathrm{QF} & :=(G \mid \exists H \in \mathfrak{F} \text { and an epimorphism from } H \text { onto } G)=\mathfrak{F}= \\
\mathrm{R}_{0} \mathfrak{F} & :=\left(G \mid \exists N_{i} \unlhd G, G / N_{i} \in \mathfrak{F}(i=1, \ldots, r) \text { with } N_{1} \cap \cdots \cap N_{r}=1\right) .
\end{aligned}
$$

Moreover, $\mathfrak{F}$ is a local formation (see $[4, \mathrm{IV}, 4.6]$ ), and thus there exists a unique map $F$ from $\mathbb{P}$ to the union of all subgroup-closed Fitting classes (including the empty class which by convention is closed under every closure operation) such that

$$
\mathfrak{F}=\bigcap_{p \in \mathbb{P}} \mathfrak{S}_{p^{\prime}} \mathfrak{S}_{p} F(p)=\bigcap_{p \in \pi(\mathfrak{F})} \mathfrak{S}_{p^{\prime}} \mathfrak{S}_{p} F(p) \cap \mathfrak{S}_{\pi(\mathfrak{F})}
$$

$F$ is integrated, that is, $F(p) \subseteq \mathfrak{F}$ for all $p \in \mathbb{P}$, and $F$ is full, that is, $F(p)=\mathfrak{S}_{p} F(p)$ for all $p \in \mathbb{P} . F$ is called the canonical local definition of $\mathfrak{F}$; further, if $\mathfrak{F}$ is of nilpotent length $r<\infty$, then $F(p)=\mathfrak{S}_{p}\left(F(p) \cap \mathfrak{N}^{r-1}\right.$ ) for all $p \in \mathbb{P}$ holds true (see [4, IV, Section 3 , Section 4] for detailed information about local formations). 


\section{Local (sub)modularity}

We recall the definition of a modular subgroup and refer the reader to [13] for further information about these subgroups.

DEFINITION 3.1. Let $G$ be a group. A subgroup $U$ of $G$ is called modular in $G$ ( $U$ $\bmod G)$ if the following conditions are satisfied:

(i) $\langle W, U\rangle \cap V=\langle W, U \cap V\rangle$ for all $W, V \leq G$ such that $W \leq V$.

(ii) $\langle W, U\rangle \cap V=\langle U, W \cap V\rangle$ for all $W, V \leq G$ such that $U \leq V$.

Evidently, each normal subgroup is a modular subgroup of $G$ but, in general, the converse does not hold true; for instance, a Sylow 2-subgroup of $S_{3}$ is modular but not normal in $S_{3}$. However, the following characterization of maximal modular subgroups, that is, subgroups of $G$ being maximal among all modular subgroups of $G$, indicates that these concepts are very close to each other.

LEMMA $3.2([13,5.1 .2])$. A subgroup $U$ of a group $G$ is a maximal modular subgroup of $G$ if and only if $U$ is a maximal normal subgroup of $G$ or $G / \operatorname{Core}_{G}(U)$ is a non-abelian group of order $p q$ (for suitable primes $p$ and $q$ ).

It is also possible to characterize arbitrary modular subgroups of a group $G$ by the structure of the corresponding quotient group $G / \operatorname{Core}_{G}(U)$ (see $[13,5.1 .14]$ ). We will only need a weak form of this statement.

THEOREM $3.3([13,5.1 .14])$. Let $G$ be a group, and let $U$ be a modular subgroup of $G$. Then $G /$ Core $_{G}(U)=S_{1} / \operatorname{Core}_{G}(U) \times \cdots \times S_{r} / \operatorname{Core}_{G}(U) \times T / \operatorname{Core}_{G}(U)$, where $r \in \mathbb{N} \cup\{0\}$, and where for all $i, j \in\{1, \ldots, r\}$ :

(a) $S_{i} / \operatorname{Core}_{G}(U) \in \mathfrak{S}_{p_{i}} \mathfrak{S}_{q_{i}}$ is a group of order $p_{i}^{n_{i}} q_{i}$, and $Z\left(S_{i} / \operatorname{Core}_{G}(U)\right)=1$ (where $q_{i}, p_{i}$ are (distinct) primes, $n_{i} \in \mathbb{N}$ ).

(b) $\left(\left|S_{i} / \operatorname{Core}_{G}(U)\right|,\left|S_{j} / \operatorname{Core}_{G}(U)\right|\right)=1=\left(\left|S_{i} / \operatorname{Core}_{G}(U)\right|, \mid T /\right.$ Core $\left._{G}(U) \mid\right)$ for $i \neq j$.

(c) $U / \operatorname{Core}_{G}(U)=Q_{1} / \operatorname{Core}_{G}(U) \times \cdots \times Q_{r} / \operatorname{Core}_{G}(U) \times(T \cap U) / \operatorname{Core}_{G}(U)$, where $Q_{i} / \operatorname{Core}_{G}(U)$ is a non-normal Sylow $q_{i}$-subgroup of $S_{i} / \operatorname{Core}_{G}(U)$.

(d) $U \cap T$ is modular and subnormal in $G$.

Definition 3.4. Let $\mathfrak{X}$ and $\mathfrak{F}$ be Fitting classes.

(a) $\mathfrak{X}$ is said to be modular in $\mathfrak{F}$ (F-modular) if $1 \neq \mathfrak{X} \subseteq \mathfrak{F}$ and an $\mathfrak{X}$-injector of $G$ is a modular subgroup of $G$ for all $G \in \mathfrak{F}$.

(b) We set $\mathrm{Y}_{\text {mod }}(\mathfrak{X})=\left(G \mid\right.$ if $V \in \operatorname{Inj}_{\mathfrak{X}}(G)$, then $V$ is modular in $\left.G\right)$. 
In [7, Theorem 1] it is proved that the concepts of $\mathfrak{S}$-modularity and $\mathfrak{S}$-normality coincide. Using Theorem 3.3 we obtain that this is valid in general.

LEMMA 3.5. Let $\mathfrak{X}$ be a non-trivial Fitting class and $G \in Y_{\text {mod }}(\mathfrak{X}) \backslash Y_{n}(\mathfrak{X})$. Then $G \times G \notin \mathrm{Y}_{\text {mod }}(\mathfrak{X})$.

PROOF. Assume to the contrary that $G \times G \in \mathrm{Y}_{\text {mod }}(\mathfrak{X})$, and let $V \in \operatorname{Inj}_{\mathfrak{X}}(G \times G)$. Then $V \geq F_{1} \times F_{2} \geq G_{\mathfrak{X}} \times G_{\mathfrak{X}}$ for suitable $F_{1}, F_{2} \in \operatorname{Inj}_{\mathfrak{X}}(G)$. Using Theorem 3.3, we obtain $\left|G / G_{\mathfrak{X}}\right|=\prod_{i=1}^{r} p_{i}^{n_{i}} q_{i} m$ and $\left|F_{1} / G_{\mathfrak{X}}\right|=\left|F_{2} / G_{\mathfrak{X}}\right|=q_{1} \cdots q_{r}$, where $r \in \mathbb{N}$, $p_{1}, \ldots, p_{r}, q_{1}, \ldots, q_{r}$ are pairwise distinct primes, $n_{i} \geq 1,\left(p_{i}, m\right)=1=\left(q_{i}, m\right)$ for all $i$, and $\left|Z\left(G / G_{\mathfrak{X}}\right)\right| \mid m$.

By $\left[4\right.$, IX, 1.1] we obtain $\left|(G \times G)_{\mathfrak{X}} /\left(G_{\mathfrak{X}} \times G_{\mathfrak{X}}\right)\right||| Z\left((G \times G) /\left(G_{\mathfrak{X}} \times G_{\mathfrak{X}}\right)\right)|| \mathrm{m}^{2}$; hence, Theorem 3.3 yields $q_{i}^{2}||(G \times G) /(G \times G)_{\mathfrak{X}} \mid$ for every $i \in\{1, \ldots, r\}$. Consequently, $q_{i} \nmid\left|V /(G \times G)_{X}\right|$. Since $F_{1} \times F_{2} \leq V$, this implies $F_{1} \times F_{2} \leq$ $(G \times G)_{\mathfrak{x}}$, a contradiction to $\left(q_{i}, m\right)=1$.

THEOREM 3.6. Let $\mathfrak{X}$ and $\mathfrak{F}$ be non-trivial Fitting classes. Then $\mathfrak{X}$ is modular in $\mathfrak{F}$ if and only if $\mathfrak{X}$ is normal in $\mathfrak{F}$.

PROOF. Obviously, every $\mathfrak{F}$-normal Fitting class is modular in $\mathfrak{F}$. Since by definition, a Fitting class is closed under products of normal subgroups, thus in particular under the formation of direct products, Lemma 3.5 yields the assertion.

In view of Theorem 3.6, we turn our attention to a weaker concept than modularity.

Definition 3.7. Let $G$ be a group. A subgroup $U$ of $G$ is called submodular in $G$ $(U \operatorname{smod} G)$ if there exists a series $U=U_{1}<U_{2}<\cdots<U_{n}=G$ of subgroups $U_{i}$ of $G$ such that $U_{i}$ is modular in $U_{i+1}$ for $i=1, \ldots, n-1$.

Obviously, this series can be chosen in such a way that $U_{i}$ is a maximal modular subgroup of $U_{i+1}$ for every $i=1, \ldots, n-1$.

Let $G$ be a group. Evidently, each modular subgroup of $G$ is submodular in $G$. The converse does not hold true in general, so for instance a Sylow 2-subgroup of $S_{3} \times S_{3}$ is submodular but not modular in $S_{3} \times S_{3}$.

Detailed analysis of submodular subgroups has been carried out by Zimmermann (see [14]), and almost all results needed here are taken from this work. The concepts of submodularity and (sub)normality, too, are very close to each other ([14, Lemma 4]):

LEMMA 3.8. Let $U$ be a submodular subgroup of a group $G$. If $K$ denotes the unique minimal normal subgroup of $U$ such that $U / K$ is abelian of square-free exponent, then $K$ is subnormal in $G$.

In particular, if $U \in \operatorname{Inj}_{\mathfrak{X}}(G)$ for a Fitting class $\mathfrak{X}$, then $U / G_{\mathfrak{X}}$ is abelian of square-free exponent. 
Groups in which all Sylow subgroups are submodular can be characterized (see $[14$, Theorem 4]). We need only a weak form of this result:

LEMMA 3.9. Let $p, q$ be primes, $q \mid p-1$, and let $G \in \mathfrak{S}_{p} \mathfrak{S}_{q}$ be such that $G / F(G)$ is an elementary abelian group. Then all Sylow subgroups of $G$ are submodular subgroups of $G$.

DEFINITION 3.10. Let $\mathfrak{X}$ and $\mathfrak{F}$ be Fitting classes.

(a) $\mathfrak{X}$ is said to be submodular in $\mathfrak{F}\left(\mathfrak{F}\right.$-submodular, $\mathfrak{F}_{\text {smod }}$-class) if $1 \neq \mathfrak{X} \subseteq \mathfrak{F}$ and an $\mathfrak{X}$-injector of $G$ is a submodular subgroup of $G$ for all $G \in \mathfrak{F}$.

(b) We set $\mathrm{Y}_{\text {smod }}(\mathfrak{X})=\left(G \mid\right.$ if $V \in \operatorname{Inj}_{\mathfrak{X}}(G)$, then $V$ is submodular in $\left.G\right)$.

Obviously, the relation of local normality implies that of local submodularity. As shown by Hauck and Kienzle (see [7, Theorem 2]) the converse also holds for $\mathfrak{F}=\mathfrak{S}$. But in general, local submodularity is a relation different from local normality. To prove this, we use a Fitting class constructed by Menth in [9], which we denote by $\mathfrak{M}(p, 3)$ (where $p$ is a prime such that $p \equiv 1 \bmod 3$ ) and which is contained in the class $\mathfrak{U}$ of all (finite) supersoluble groups. We will not present the (complex) definition of this class, but only the following statements used here $([9,4.2,4.3])$ :

THEOREM 3.11. Let $\mathfrak{M}(p, 3)$ be as described in [9].

(a) $\mathfrak{M}(p, 3)$ is a Fitting class such that $\mathfrak{S}_{p} \times \mathfrak{S}_{3} \subset \mathfrak{M}(p, 3) \subseteq \mathfrak{S}_{p} \mathfrak{S}_{3} \cap \mathfrak{U}$

(b) If $G \in \mathfrak{M}(p, 3)$, then $G / F(G)$ is an elementary abelian 3-group.

PROPOSITION 3.12. Let $\mathfrak{M}(p, 3)$ be as described in [9]. Then $\mathfrak{N S}_{3}$ is submodular, but not normal in $\mathfrak{N M}(p, 3)$.

ProOF. (1): Let $G$ be a group contained in $\mathfrak{N M}(p, 3)$. Then $F(G) P_{3}$ is an $\mathfrak{N S}_{3}$ injector of $G$ (where $P_{3} \in \operatorname{Syl}_{3}(G)$ ). Let $T$ be an $\mathfrak{N}$-injector of $G_{\mathfrak{L}_{3}(\mathfrak{N})}$, where $\mathfrak{L}_{3}(\mathfrak{N})$ denotes the Fitting class of all groups $H$ such that an $\mathfrak{N}$-injector of $H$ contains a Sylow 3-subgroup of $H$ (see [4, IX, Section 1]). According to [4, IX, 1.22], $T P_{3} \in \operatorname{Inj}_{\mathfrak{N G}_{3}}(G)$ for a suitable Sylow 3-subgroup $P_{3}$ of $G$. Set $N=G_{\mathcal{L}_{3}(\mathfrak{N})}$. By [4, IX, 4.12] $T=\prod T_{q}$ where $T_{q} \in \operatorname{Syl}_{q}\left(C_{N}\left(O_{q^{\prime}}(F(N))\right)\right)$. In particular, $T_{p}$ is a normal subgroup of $F(G) T_{p}$, and consequently $T_{p}=O_{p}(G)$. Obviously, $O_{q}(G)=T_{q}$ for $q \neq p, 3$. Hence we obtain $T P_{3}=F(G) P_{3}$, and the proof is complete.

(2): $\mathfrak{N S}_{3}$ is submodular in $\mathfrak{N M}(p, 3)$ : Let the notation be as in (1). It follows from Theorem 3.11 and Lemma 3.9 that $F(G) P_{3} / F(G)$ is a submodular subgroup of $G / F(G)$; thus (1) yields the assertion.

(3): $\mathfrak{N M}(p, 3) \notin \mathrm{Y}_{\mathrm{n}}\left(\mathfrak{N S}_{3}\right)$ : Suppose that $\mathfrak{N M}(p, 3) \subseteq \mathrm{Y}_{\mathrm{n}}\left(\mathfrak{N S _ { 3 }}\right)$. By Theorem 3.11 there exists a group $G \in \mathfrak{M}(p, 3) \backslash \mathfrak{S}_{p} \times \mathfrak{S}_{3}$. Let $q$ be a prime $\neq p, 3$. 
Then (1) implies $G \cong Z_{q} 2 G / F\left(Z_{q} 2 G\right) \in \mathfrak{S}_{3} \mathfrak{S}_{p} \cap \mathfrak{S}_{p} \mathfrak{S}_{3}$, a contradiction to the choice of $G$.

In the treatment of locally normal Fitting classes it is possible to confine oneself to the case that both classes are Lockett classes, thus to classes which are easier to handle than arbitrary Fitting classes (see $[6,4.8]$ or $[4, X, 3.3]$ ). The next result shows that this remains valid for locally submodular Fitting classes.

THEOREM 3.13. Let $\mathfrak{X}$ and $\mathfrak{F}$ be Fitting classes. Then the following statements are equivalent:

(i) $\mathfrak{X}$ is submodular in $\mathfrak{F}$.

(ii) $\mathfrak{X}$ is submodular in $\mathfrak{F}^{*}$.

(iii) $\mathfrak{X}^{*}$ is submodular in $\mathfrak{F}^{*}$.

PROOF. (i) $\Rightarrow$ (iii): Let $G \in \mathfrak{F}^{*}, V \in \operatorname{Inj}_{\mathfrak{x}^{*}}(G)$. According to Theorem 2.1 and Theorem 2.2 the group $(V \times V)_{\mathfrak{X}}$ is an $\mathfrak{X}$-injector of $G \times G$ and by assumption we obtain that $(V \times V)_{\mathfrak{X}} \cap(G \times G)_{\mathfrak{F}}$ is submodular in $(G \times G)_{\mathfrak{F}}$. Moreover, submodularity is invariant under epimorphisms, hence Theorem 2.1 yields that $V$ is a submodular subgroup of $G$ and the proof is complete. (iii) $\Rightarrow$ (ii): Since by definition and Theorem 2.1 the class $Y_{\text {smod }}\left(\mathfrak{X}^{*}\right)$ is contained in $Y_{\text {smod }}(\mathfrak{X})$, the assertion follows. (ii) $\Rightarrow$ (i): Obvious.

Note that this proof can be easily transferred to every embedding property e of injectors such that $Y_{e}(\mathfrak{X})$ is closed under normal subgroups, $e$ is invariant under epimorphisms and such that the class $Y_{e}\left(\mathfrak{X}^{*}\right)$ is contained in the class $Y_{e}(\mathfrak{X})$.

Hauck has shown in $[6,3.2]$ that the class $Y_{n}(X)$, which obviously is closed under taking subnormal subgroups, in general fails to be closed under products of normal subgroups. Consequently the question on the existence of a unique maximal Fitting class contained in $Y_{n}(\mathfrak{X})$ is open in general.

Unfortunately, the same is valid for the class $Y_{\text {smod }}(\mathfrak{X})$ :

PROPOSITION 3.14. Let $\mathfrak{X}$ be a Fitting class. Then $\mathrm{Y}_{\text {smod }}(\mathfrak{X})$ need not be closed under forming normal products.

PROOF. Let $p$ and $q$ be prime numbers such that $p \mid q-1$. Set $H=Z_{p} \imath Z_{p}=$ $H_{1} H_{2}$ where $H_{1} \cong Z_{p}^{*}$ and $H_{2} \cong Z_{p}$. Consider the group $G=Z_{q} 2 H$. Then $G=\left\langle Z_{q}^{*} H_{1}, Z_{q}^{*} H_{2}\right\rangle$, and $Z_{q}^{*} H_{1}$ and $Z_{q}^{*} H_{2}$ are subnormal $\mathrm{Y}_{\text {smod }}\left(\mathfrak{S}_{p}\right)$-subgroups of $G$ according to Lemma 3.9. But evidently, $O_{p}(G)=1$ and an $\mathfrak{S}_{p}$-injector of $G$ is a non-abelian subgroup of $G$. By Lemma 3.8, this implies $G \notin \mathrm{Y}_{\text {smod }}\left(\mathfrak{S}_{p}\right)$.

PROPOSITION 3.15. Let $\mathfrak{X}$ be a Lockett class. Then $\mathrm{Y}_{\text {smod }}(\mathfrak{X})$ is closed under forming direct products. 
PROOF. Evidently, it is sufficient to prove that $G_{1} \times G_{2} \in \mathrm{Y}_{\text {smod }}(\mathfrak{X})$ provided that $G_{1}, G_{2} \in \mathrm{Y}_{\text {smod }}(\mathfrak{X})$. Thus, let $G_{1}, G_{2}$ be groups belonging to $\mathrm{Y}_{\text {smod }}(\mathfrak{X})$ and set $G=G_{1} \times G_{2}$. Theorem 2.2 states that $V_{1} \times V_{2} \in \operatorname{Inj}_{\mathfrak{X}}(G)$ where $V_{i} \in \operatorname{Inj}_{\mathfrak{X}}\left(G_{i}\right)$, and that each $\mathfrak{X}$-injector of $G$ is of this form $(i=1,2) . V_{i}$ smod $G_{i}$, consequently there exists a series $V_{i}=D_{0}^{i} \leq D_{1}^{i} \leq \cdots \leq D_{n_{i}}^{i}=G_{i}$ such that $D_{j}^{i}$ is a maximal modular subgroup of $D_{j+1}^{i}$ for all $j=1, \ldots, n_{i}-1 ; i=1,2$. We assume that $n_{1} \leq n_{2}$ and consider the series $V_{1} \times V_{2}=D_{0}^{1} \times D_{0}^{2} \leq D_{0}^{1} \times D_{1}^{2} \leq D_{1}^{1} \times D_{1}^{2} \leq \cdots \leq D_{n_{1}}^{1} \times D_{n_{1}}^{2} \leq$ $D_{n_{1}}^{1} \times D_{n_{1}+1}^{2} \leq D_{n_{1}}^{1} \times D_{n_{1}+2}^{2} \leq \cdots \leq D_{n_{1}}^{1} \times D_{n_{2}}^{2}=G_{1} \times G_{2}$. Now, repeated application of Lemma 3.2 yields the assertion.

Let $\mathfrak{F}$ be a non-trivial Fitting class, and let $\mathfrak{X}_{i}, i \in I$, denote $\mathfrak{F}$-submodular Fitting classes. Whether or not $\mathfrak{F}$ is contained in $\mathrm{Y}_{\text {smod }}\left(\bigcap_{i \in I} \mathfrak{X}_{i}\right)$-and thus in particular whether or not there exists a unique minimal $\mathfrak{F}$-submodular Fitting class-is an open question. It is open even in the case when $\mathfrak{F}$ is a Fischer class (or, stronger, when $\mathfrak{F}$ is an subgroup-closed Fitting class). Compared to local normality, in this situation it seems to be harder to describe the structure of a minimal counterexample for two reasons. On the one hand, submodular subgroups do not, in general, form a lattice. On the other hand, there is nothing known, in general, about the relation between $\mathfrak{X}_{i}$-injectors and $\bigcap_{i \in I} \mathfrak{X}_{i}$-injectors of a group $G$.

Nevertheless, in some important cases it is possible to obtain a positive answer to the above mentioned question. This comes mainly from the validity of the following lemma.

LEMMA 3.16. Let $\mathfrak{X}$ and $\mathfrak{F}$ be Lockett classes such that $\mathfrak{X}$ is submodular in $\mathfrak{F}$. Further let $G$ be a group contained in $\mathfrak{X}$ and $p, q(p \neq q)$ be primes such that $G: Z_{p} \in \mathfrak{X}$ and $G \geq Z_{q}, Z_{p} \in \mathfrak{F}$. Then $G \geq Z_{q} \in \mathfrak{X}$.

In particular, if $\mathfrak{G}$ is a Lockett class such that $\mathfrak{G S}_{p} \subseteq \mathfrak{X}$ and $\mathfrak{G S}_{p} \mathfrak{S}_{q} \mathfrak{S}_{p} \subseteq \mathfrak{F}$, then $\mathfrak{G S}_{p} \mathfrak{S}_{q} \subseteq \mathfrak{X}$.

PROOF. Let $P$ denote a non-abelian $p$-group. According to [4, X, 2.7], $G: Z_{p} \in \mathfrak{X}$ implies that $G \geq P$ belongs to $\mathfrak{X}$. Assume that $G \geq Z_{q} \notin \mathfrak{X}$. Then $[4, X, 2.1]$ yields $\left(G \prec Z_{q} \prec P\right)_{\mathfrak{X}}=\left(G^{*}\right)^{*}$. Thus by $[4, \mathrm{IX}, 1.6]$ we obtain $\left(G^{*}\right)^{*} P \in \operatorname{Inj}_{\mathfrak{X}}\left(G \imath Z_{q} \prec P\right)$, a contradiction to Lemma 3.8. The final assertion follows from [4, X, 2.13]

Using a corresponding lemma we have shown in $[10,1.3,2.3,2.7]$ that for the important Fitting classes $\mathfrak{N}^{i+1}, i \in \mathbb{N}$, and $\mathfrak{S}_{p_{1}} \ldots \mathfrak{S}_{p_{r}}, p_{i}$ primes, and for Fitting classes satisfying a certain extension property with respect to wreath products a unique minimal $\mathfrak{F}$-normal Fitting class exists and furthermore can be described explicitly. Essentially analogously to $[10,2.7]$ —but with more technical effort-the existence and an explicit description of a unique minimal $\mathfrak{F}$-normal Fitting class can be shown for Fitting classes $\mathfrak{F}=\mathfrak{S}_{\pi_{1}} \ldots \mathfrak{S}_{\pi_{r}}$, where $\pi_{1}, \ldots, \pi_{r}$ are arbitrary sets of primes ([11, 
3.1.18]). Since, as shown above, local submodularity too behaves nicely with respect to regular wreath products, we obtain using essentially the same arguments as in case of local normality:

THEOREM 3.17. (a) Let $\mathfrak{X}$ be a non-trivial Fitting class, $i \in \mathbb{N}$. Then $\mathfrak{X}$ is an $\mathfrak{N}^{i+1}$-submodular Fitting class if and only if $\mathfrak{N}^{i} \subseteq \mathfrak{X}^{*} \subseteq \mathfrak{N}^{i+1}$.

In particular, there exists a unique minimal $\mathfrak{N}^{i+1}$-submodular Fitting class, namely $\left(\mathfrak{N}^{i}\right)_{*}$.

(b) Let $\mathfrak{K}$ be an $\mathrm{S}_{\mathrm{n}}$-closed class of groups such that $G \geq Z_{p} \in \mathfrak{K}$ for all $G \in \mathfrak{K}$ and for all $p \in \pi(\mathfrak{K})$. Set $\mathfrak{F}=\operatorname{Fit}(\mathfrak{K})^{*}$, the smallest Lockett class containing $\mathfrak{K}$. Then a Fitting class $\mathfrak{X}$ is submodular in $\mathfrak{F}$ if and only if $\mathfrak{X}^{*}=\mathfrak{F}$.

In particular, there exists a unique minimal $\mathfrak{F}$-submodular Fitting class, namely $\mathfrak{F}_{*}$.

(c) Let $\pi_{1}, \ldots, \pi_{r}$ be arbitrary sets of primes and set $\mathfrak{F}=\mathfrak{S}_{\pi_{1}} \ldots \mathfrak{S}_{\pi_{r}}$. Then there exists a unique minimal $\mathfrak{F}$-submodular Fitting class, which can be explicitly given as described in $[11,3.1 .18]$.

In particular, if $\pi_{1}, \ldots, \pi_{r}$ are sets of primes such that $\pi_{1} \cap \cdots \cap \pi_{r} \neq \emptyset$, then $\mathfrak{F}_{*}$ is the unique minimal $\mathfrak{F}$-submodular Fitting class.

In particular, for all classes $\mathfrak{F}$ described above the unique minimal $\mathfrak{F}$-submodular Fitting class coincides with the unique minimal $\mathfrak{F}$-normal Fitting class. Furthermore, for classes $\mathfrak{F}$ as described in (a) and (b) a Fitting class $\mathfrak{X}$ is submodular in $\mathfrak{F}$ if and only if $\mathfrak{X}$ is normal in $\mathfrak{F}$.

Closing this section we prove that the concepts of local submodularity and local normality between Fitting classes coincide provided that both classes are subgroupclosed Fitting classes. Whether or not it is sufficient for this fact to require the subgroup closure of the larger class, remains an open question.

LEMMA 3.18. Let $\mathfrak{X}$ be a Fitting class and set $\pi=\pi(\mathfrak{X})$. If $\mathfrak{F}$ is a subgroup-closed Fitting class contained in $\mathrm{Y}_{\text {smod }}(\mathfrak{X})$, then $\mathfrak{F} \subseteq \mathfrak{S}_{\pi} \mathfrak{S}_{\pi^{\prime}}$.

Proof. Assume not. Let $G$ be a group of minimal order contained in $\mathfrak{F} \backslash \mathfrak{S}_{\pi} \mathfrak{S}_{\pi^{\prime}}$. Then $G$ has a unique maximal normal subgroup $N$, and a unique minimal normal subgroup $M$, and $N$ and $G / M$ belong to $\mathfrak{S}_{\pi} \mathfrak{S}_{\pi^{\prime}}$. Moreover, $O_{\pi}(G)=1$ and $O^{\pi^{\prime}}(G)=$ $G$. Consequently $[4, \mathrm{XI}, 2.4,2.5]$ is appliable and we obtain $\mathfrak{S}_{q} \mathfrak{S}_{p} \subseteq \mathfrak{F} \subseteq \mathrm{Y}_{\text {smod }}(\mathfrak{X})$ for primes $q \in \pi^{\prime}$ and $p \in \pi$, a contradiction to Lemma 3.8.

THEOREM 3.19. Let $\mathfrak{X}, \mathfrak{F}$ be subgroup-closed Fitting classes such that $\mathfrak{F} \subseteq \mathrm{Y}_{\text {smod }}(\mathfrak{X})$. Then $\mathfrak{F} \subseteq \mathrm{Y}_{\mathrm{n}}(\mathfrak{X})$.

In particular, $\mathfrak{X}$ is submodular in $\mathfrak{F}$ if and only if $\mathfrak{X}$ is $\mathfrak{F}$-normal. 
Proof. Since each $G \in \mathfrak{F}$ is of finite nilpotent length, it is sufficient to prove the assertion for a subgroup-closed Fitting class $\mathfrak{F}$ of bounded nilpotent length. This will be done by induction on $r:=l(\mathfrak{F})$. The cases $r=0,1$ are obvious.

$r>1$ : According to Lemma 3.18, we may assume that $\pi(\mathfrak{F}) \subseteq \pi(\mathfrak{X})$. Let $X$ and $F$ denote the canonical local definitions belonging to $\mathfrak{X}$ and $\mathfrak{F}$, respectively. Then analogously to the proof of $[12,2.7]$, we obtain $F(p) \cap \mathfrak{N}^{r-1} \subseteq F(p) \subseteq \mathrm{Y}_{\text {smod }}(X(p))$ for every $p \in \pi$. By the inductive hypothesis this implies $F(p) \cap \mathfrak{N}^{r-1} \subseteq \mathrm{Y}_{\mathrm{n}}(X(p))$, and $[12,2.5]$ yields $F(p)=\mathfrak{S}_{p}\left(F(p) \cap \mathfrak{N}^{r-1}\right) \subseteq \mathrm{Y}_{\mathrm{n}}(X(p))$. [12, 2.7] completes the proof.

\section{Local normal embedding}

We recall the definition of a normally embedded subgroup and refer the reader to [4, I, Section 7] for further information about these subgroups.

DEFINITION 4.1. Let $G$ be a group and $U$ be a subgroup of $G$.

(a) Let $p$ be a prime. $U$ is called $p$-normally embedded in $G$ ( $U p$-ne $G$ ) if a Sylow $p$-subgroup $U_{p}$ of $U$ is a Sylow $p$-subgroup of some normal subgroup of $G$, that is, $U_{p} \in \operatorname{Syl}_{p}\left(\left\langle U_{p}^{G}\right\rangle\right)$.

(b) $U$ is called normally embedded in $G(U$ ne $G)$ if $U$ is $p$-normally embedded in $G$ for all primes $p$.

Typical examples of normally embedded subgroups of a group $G$ are the Hall subgroups of a normal subgroup of $G$.

DefINITION 4.2. Let $\mathfrak{X}$ and $\mathfrak{F}$ be Fitting classes.

(a) $\mathfrak{X}$ is called normally embedded in $\mathfrak{F}$ ( $\mathfrak{F}$-normally embedded, $\mathfrak{F}_{\text {ne }}$-class), if $1 \neq$ $\mathfrak{X} \subseteq \mathfrak{F}$ and an $\mathfrak{X}$-injector of $G$ is normally embedded in $G$ for all $G \in \mathfrak{F}$.

(b) We set $\mathrm{Y}_{\text {ne }}(\mathfrak{X})=\left(G \mid\right.$ if $V \in \operatorname{Inj}_{\mathfrak{X}}(G)$, then $V$ ne $\left.G\right)$.

S-normally embedded Fitting classes have been studied in detail by Lockett [8] and Doerk and Porta [5] (see [4, IX, Section 3]). By [4, IX, 3.4(a)], each Fischer classthus in particular each subgroup-closed Fitting class-is an $\mathfrak{S}$-normally embedded Fitting class, and according to $[4, \mathrm{IX}, 2.9,3.7]$, the class $\mathfrak{Z}^{3}=\left(G \mid \operatorname{Soc}_{3}(G) \leq Z(G)\right)$ is a Lockett class which is not normally embedded in $\mathfrak{S}$.

Recall the following properties of normally embedded injectors (which are due to Lockett, see $[8$, proof of 3.3.1, 3.3.6]).

LEMMA 4.3. Let $\mathfrak{X}, \mathfrak{Y}$ and $\mathfrak{F}$ be Fitting classes, let $G$ be a group and $p$ a prime. 
(a) If $N \unlhd G$ and $V \cap N p$-ne $N$, then $V \cap N p$-ne $G$ where $V \in \operatorname{Inj}_{\mathfrak{X}}(G)$.

(b) If $V \in \operatorname{Inj}_{\mathfrak{Y}}(G)$ and $W \in \operatorname{Inj}_{\mathfrak{X}}(V)$ such that $V p$-ne $G$ and $W p$-ne $V$, then $W$ $p$-ne $G$.

In particular, $\mathrm{Y}_{\mathrm{ne}}\left(\mathfrak{X}^{*}\right) \subseteq \mathrm{Y}_{\mathrm{ne}}(\mathfrak{X})$.

Since obviously $Y_{n e}(\mathfrak{X})=S_{n} Y_{n e}(\mathfrak{X})$ and normal embedding is a property invariant under epimorphisms, we obtain that this relation too is a relation of the corresponding Lockett sections.

REMARK 4.4. Let $\mathfrak{X}$ and $\mathfrak{F}$ be Fitting classes. Then the following statements are equivalent:

(i) $\mathfrak{X}$ is normally embedded in $\mathfrak{F}$.

(ii) $\mathfrak{X}$ is normally embedded in $\mathfrak{F}^{*}$.

(iii) $\mathfrak{X}^{*}$ is normally embedded in $\mathfrak{F}^{*}$.

Evidently, the class $Y_{n e}(\mathfrak{X})$ is closed under forming direct products provided that $\mathfrak{X}=\mathfrak{X}^{*}$. But in general, $Y_{n e}(\mathfrak{X})$ fails to be a Fitting class:

Proposition 4.5. $\mathfrak{X}=\mathfrak{Z}^{3}=\left(G \mid \operatorname{Soc}_{3}(G) \leq Z(G)\right)$ is a Fitting class such that $\mathrm{Y}_{\text {ne }}(\mathfrak{X}) \neq \mathrm{N}_{0} \mathrm{Y}_{\text {ne }}(\mathfrak{X})$.

ProOF. Assume to the contrary that $Y_{n e}(\mathfrak{X})=\mathrm{N}_{0} Y_{\text {ne }}(\mathfrak{X})$; then Remark 4.4 yields $\mathrm{Y}_{\mathrm{ne}}(\mathfrak{X})=\mathrm{Y}_{\mathrm{ne}}(\mathfrak{X})^{*}$. We will prove that this implies $G \geq Z_{p} \in \mathrm{Y}_{\mathrm{ne}}(\mathfrak{X})$ for every $G \in \mathrm{Y}_{\text {ne }}(\mathfrak{X})$ and every prime $p$. Then $[4, \mathrm{X}, 3.7]$ yields $\mathrm{Y}_{\mathrm{ne}}(\mathfrak{X})=\mathfrak{S}$, a contradiction.

By a result proved independently by Lockett and Frantz (see [4, IX, 4.19]), the radicals and injectors of $\mathfrak{X}$ are known: $G_{\mathfrak{X}}=C_{G}\left(\operatorname{Soc}_{3}(G)\right)$ and $\operatorname{Inj}_{\mathfrak{X}}(G)=$ $\left\{C_{G}\left(C_{\mathrm{Soc}_{3}\left(G_{x}\right)}\left(G_{3}\right)\right) \mid G_{3} \in \operatorname{Syl}_{3}(G)\right\}$ for every group $G$.

Let $G$ be a group contained in $\mathrm{Y}_{\mathrm{ne}}(\mathfrak{X})$, and let $p$ be a prime; then $G$ ? $Z_{p} \in \mathrm{Y}_{\mathrm{n}}(\mathfrak{X})$ :

$p=3$ : Let $V$ be an $\mathfrak{X}$-injector of $G$ ? $Z_{p}$. If $V \leq G^{*}$, there is nothing to prove. Thus we may assume that $V \npreceq G^{*}$. Then $V \cong F^{*} Z_{p}$ for a suitable $F \in \operatorname{Inj}_{\mathfrak{X}}(G)$. If $q \neq p$, then evidently $V q$-ne $G$ ? $Z_{p}$. Since according to the above mentioned description of $V$, a Sylow 3-subgroup of $V$ is a Sylow 3-subgroup of $G$ ? $Z_{p}$ as well, we obtain that $V$ 3-ne $G \geq Z_{p}$.

$p \neq 3$ : Put $H:=G \imath Z_{p}$ and assume that $H \notin \mathrm{Y}_{\mathrm{pe}}(\mathfrak{X})$. Let $V \in \operatorname{Inj}_{\mathfrak{X}}(H)$. Since $p \neq 3, G_{3}^{*}=H_{3} \in \operatorname{Syl}_{3}(H)$ (where $G_{3} \in \operatorname{Syl}_{3}(G)$ ). Since $G \notin \mathfrak{X}$, it follows from [4, $\mathrm{X}, 2.1]$ that $H_{\mathfrak{X}}=G_{\mathfrak{X}}^{*} \leq G^{*}$, and consequently that $G^{*} \geq \operatorname{Soc}_{3}\left(H_{\mathfrak{X}}\right) \geq\left(\operatorname{Soc}_{3}\left(G_{\mathfrak{X}}\right)\right)^{*}$. If $V=C_{H}\left(C_{\mathrm{Soc}_{3}\left(H_{x}\right)}\left(G_{3}^{*}\right)\right) \notin G^{*}$, then there exists an element

$$
\begin{aligned}
\left(x_{1}, \ldots, x_{p} ; z\right) & \in C_{H}\left(C_{\mathrm{Soc}_{3}\left(H_{x}\right)}\left(G_{3}^{*}\right)\right) \leq C_{H}\left(C_{\left(\mathrm{Soc}_{3}\left(G_{x}\right)\right)^{*}}\left(G_{3}^{*}\right)\right) \\
& =C_{H}\left(\left(C_{\mathrm{Soc}_{3}\left(G_{x}\right)}\left(G_{3}\right)\right)^{*}\right)
\end{aligned}
$$


such that $z \neq 1$. By construction of the regular wreath product this implies $C_{\mathrm{Soc}_{3}\left(G_{x}\right)}\left(G_{3}\right)=1$, a contradiction.

Hence we obtain $V \leq G^{*}$, and Lemma 4.3 yields $V$ ne $H$, a final contradiction.

It is an open question, whether or not $Y_{n e}(\mathfrak{X})=N_{0} Y_{\text {ne }}(\mathfrak{X})$ implies that $\mathfrak{X}$ is normally embedded in $\mathfrak{S}$ in the case that $\mathfrak{X}$ is an arbitrary Lockett class. It is open as well whether the intersection of $\mathfrak{F}_{n e}$-classes is again an $\mathfrak{F}_{\text {ne }}$-class (provided that it is non-trivial). This question is open even in the case that $\mathfrak{F}=\mathfrak{S}$.

\section{Local permutability}

Recall that a Hall system of a group $G$ is a set $\Sigma$ of Hall subgroups of $G$ such that for each set of primes $\pi \subseteq \pi(G), \Sigma$ contains exactly one Hall $\pi$-subgroup of $G$, and that $H, K \in \Sigma$ implies $H K=K H$ (that is, $H$ and $K$ permute). Recall further that to each Hall system $\Sigma$ there exists a unique corresponding Sylow basis-a set $B_{\Sigma}$ of pairwise permutable Sylow $p$-subgroups of $G$, exactly one for each $p \in \pi(G)$, together with the identity group (see $[4, I, 4.8]$ ).

We recall the definition of a system permutable subgroup and refer the reader to $[4$, I, Section 4] for further information about these subgroups.

DEFINITION 5.1. Let $G$ be a group and $U$ a subgroup of $G$ and let $\Sigma$ denote a Hall system of $G$. Then $U$ is called $\Sigma$-permutable if $U H=H U$ for all $H \in \Sigma$.

We say that $U$ is system permutable in $G(U$ sp $G)$ if there exists a Hall system $\Sigma$ of $G$ such that $U$ is $\Sigma$-permutable.

To obtain $\Sigma$-permutability of a subgroup $U$ of $G$, it is sufficient to require that $U$ permutes with the corresponding Sylow basis, that is, $U$ is $\Sigma$-permutable if and only if $U H=H U$ for every $H \in B_{\Sigma}([4,1,4.26])$.

Obviously, each normal subgroup of a group $G$ is system permutable in $G$ and according to $[4, I, 7.10]$, each normally embedded subgroup, too, is a system permutable subgroup of $G$.

DEFINITION 5.2. Let $\mathfrak{X}$ and $\mathfrak{F}$ be Fitting classes.

(a) $\mathfrak{X}$ is called permutable in $\mathfrak{F}$ (F-permutable, $\mathfrak{F}_{\mathrm{p}}$-class), if $1 \neq \mathfrak{X} \subseteq \mathfrak{F}$ and an $\mathfrak{X}$-injector of $G$ is system permutable in $G$ for all $G \in \mathfrak{F}$.

(b) We set $\mathrm{Y}_{\mathrm{p}}(\mathfrak{X})=\left(G \mid\right.$ if $V \in \operatorname{Inj}_{\mathfrak{X}}(G)$, then $V$ sp $\left.G\right)$.

Since a normally embedded subgroup of a group $G$ is system permutable in $G$, every $\mathfrak{F}$-normally embedded Fitting class is permutable in $\mathfrak{F}$. (The converse does not hold in general, as shown in [4, IX, 3.15].) In particular, every Fischer class-and consequently every subgroup-closed Fitting class-is an $\mathfrak{S}$-permutable Fitting class. 
In 1972, Dark published an example of a Fitting class which is not permutable in $\mathfrak{S}$ (see [4, IX, 5.19]).

In 1980 , Doerk and Porta proved that a Fitting class $\mathfrak{F}$ is permutable in $\mathfrak{S}$ precisely when $\mathfrak{F}^{*}$ is $\mathfrak{S}$-permutable (see $[4, X, 1.39]$ ), and using essentially the same arguments we obtain that $Y_{p}\left(\mathfrak{X}^{*}\right) \subseteq Y_{p}(\mathfrak{X})$ for an arbitrary Fitting class $\mathfrak{X}$. Since further $Y_{p}(\mathfrak{X})=$ $s_{n} Y_{p}(\mathfrak{X})$ and system permutability is a property invariant under epimorphisms, we conclude:

REMARK 5.3. Let $\mathfrak{X}$ and $\mathfrak{F}$ be Fitting classes. Then the following statements are equivalent:

(i) $\mathfrak{X}$ is permutable in $\mathfrak{F}$.

(ii) $\mathfrak{X}$ is permutable in $\mathfrak{F}^{*}$.

(iii) $\mathfrak{X}^{*}$ is permutable in $\mathfrak{F}^{*}$.

Evidently, the class $Y_{p}(\mathfrak{X})$ is closed under under forming direct products provided that $\mathfrak{X}=\mathfrak{X}^{*}$. But, in general, $Y_{p}(\mathfrak{X})$ fails to be a Fitting class:

Proposition 5.4. Let $\mathfrak{X}$ be a Lockett class, let $G \in Y_{\mathrm{p}}(\mathfrak{X})$ and let $p$ be a prime. Then $G: Z_{p} \in \mathrm{Y}_{\mathrm{p}}(\mathfrak{X})$.

In particular, $\mathrm{Y}_{\mathrm{p}}(\mathfrak{X})=\mathrm{N}_{0} \mathrm{Y}_{\mathrm{p}}(\mathfrak{X})$ for an arbitrary Fitting class $\mathfrak{X}$ if and only if $\mathfrak{X}$ is permutable in $\mathfrak{S}$.

PROOF. Suppose that the first assertion holds. Then, by Remark 5.3, $Y_{p}(\mathfrak{X})=$ $Y_{p}(\mathfrak{X})^{*}=Y_{p}\left(\mathfrak{X}^{*}\right)$. Hence we may assume that $\mathfrak{X}=\mathfrak{X}^{*}$. [4, $\left.X, 3.7\right]$ implies that $Y_{p}(\mathfrak{X})=\mathfrak{S}$, and the additional remark is valid as well.

To prove the first assertion we put $H=G \imath Z_{p}$. Let $F$ be an $\mathfrak{X}$-injector of $H$. If $F \leq G^{*}$, then it follows by the choice of $G$ and by the construction of the regular wreath product that $F$ is system permutable in $H$, and we are finished. Thus we may assume that $F \notin G^{*}$ and consequently that $F=V^{*} Z_{p}$ for some $V \in \operatorname{Inj}_{\mathfrak{X}}(G)$. By assumption, $V^{*}$ is a system permutable subgroup of $G^{*}$. Let $\Sigma=\left\{G_{\pi}^{*} \mid \pi \subseteq \pi(G)\right\}$ denote a corresponding Hall system of $G^{*}$. Then by construction of the regular wreath product, $Z_{p} \leq N_{H}\left(U^{*}\right)$ for every subgroup $U$ of $G$. Consequently, $F$ permutes with $G_{\pi}^{*} Z_{p} \in \operatorname{Hall}_{\pi}(H)$ where $\pi$ is a set of primes containing $p$. If $\pi \subseteq \mathbb{P} \backslash\{p\}$, then $G_{\pi}^{*} \in \operatorname{Hall}_{\pi}(H)$, and $F$ permutes with $G_{\pi}^{*}$. Observe further that $\Sigma_{0}:=\left\{G_{\pi}^{*} Z_{p} \mid \pi \subseteq\right.$ $\mathbb{P}, p \in \pi\} \cup\left\{G_{\pi}^{*} \mid \pi \subseteq \mathbb{P}, p \notin \pi\right\}$ forms a Hall system of $H$; hence the proof is complete.

Thus also the question on a unique maximal Fitting class contained in $Y_{p}(\mathfrak{X})$ is open in general. The (non-trivial) intersection of $\mathfrak{F}$-permutable Fitting classes is not, in general, $\mathfrak{F}$-permutable, not even in the case that $\mathfrak{F}=\mathfrak{S}$ (see $[4, \mathrm{IX}, 3.14]$ ). 


\section{Acknowledgement}

The results of this paper were part of the author's doctoral thesis. The author expresses her gratitude to Professor P. Hauck for many helpful discussions and for the kind supervision of her thesis.

\section{References}

[1] D. Blessenohl and W. Gaschütz, 'Über normale Schunck- und Fittingklassen', Math. Z. 118(1970), 1-8.

[2] R. A. Bryce and J. Cossey, 'Fitting formations of finite soluble groups', Math. Z. 127 (1972), 217-223.

[3] _ - 'Subgroup closed Fitting classes are formations', Math. Proc. Cambridge Philos. Soc. 91 (1982), 225-258.

[4] K. Doerk and T. Hawkes, Finite soluble groups (de Gruyter, Berlin, 1992).

[5] K. Doerk and M. Porta, 'Über Vertauschbarkeit, normale Einbettung und Dominanz bei Fittingklassen endlicher aufösbarer Gruppen’, Arch. Math. 35 (1980), 319-327.

[6] P. Hauck, Zur Theorie der Fittingklassen endlicher auflösbarer Gruppen (Dissertation, Mainz, 1977).

[7] P. Hauck and R. Kienzle, 'Modular Fitting functors in finite groups', Bull. Austral. Math. Soc. 36 (1987), 475-483.

[8] F. P. Lockett, On the theory of Fitting classes of finite soluble groups (Ph.D. Thesis, Warwick, 1971).

[9] M. Menth, 'A family of Fitting classes of supersoluble groups', Math. Proc. Cambridge Philos. Soc. 18 (1995), 49-57.

[10] S. Reifferscheid, 'On $\mathfrak{F}$-normal Fitting classes of finite soluble groups', Arch. Math. 75 (2000), 164-172.

[11] - On the theory of Fitting classes of finite soluble groups (Dissertation, Tübingen, 2001).

[12] __ ' 'On locally normal Fitting classes of finite soluble groups', J. Algebra 261 (2003), 186-206.

[13] R. Schmidt, Subgroup lattices of groups (de Gruyter, Berlin, 1992).

[14] I. Zimmermann, 'Submodular subgroups in finite groups', Math. Z. 202 (1989), 545-557.

Wilhelm-Schickard Institut für Informatik

Universität Tübingen

Sand 14

72076 Tübingen

Germany

e-mail: reifferscheid@informatik.uni-tuebingen.de 
